\title{
An HI Study of Two Barred Spiral Galaxies
}

\author{
E. M. Moore
}

Department of Astronomy, Boston University, Boston, MA 02215

\section{S. T. Gottesman}

Department of Astronomy, University of Florida, Gainesville, FL 32611

\section{Introduction}

We have made HI observations of an early type, NGC 1398 (SBab(r)I), and a late type barred system, NGC 3319 (SBc(s)II.4). NGC 1398 is a beautifully symmetric galaxy with a bright stellar bulge, bar and inner ring and tightly wound spiral arms (also classified as an $R_{1} R_{2}^{\prime}$ double outer pseudoring). In HI the spiral structure is strong but the center shows a sharp depletion. In contrast, NGC 3319 has an asymmetric appearance and irregular spiral structure. It is rich in its central HI with a gas bar and spiral arms, both of which are highlighted by prominent HII regions. We attempt to determine the pattern speeds of the galaxies and to explore the differences between early and late type systems.

\section{NGC 1398}

NGC 1398 was observed at the Very Large Array (VLA) in the DnC and CnB configurations with a spatial resolution ranging from $15^{\prime \prime}$ to 60 "and a channel separation of $20.7 \mathrm{~km} \mathrm{~s}^{-1}$. Details of the observations are given in Moore \& Gottesman (1995a). A global HI image (Figure 1) shows a severe depletion at the center and high density, segmented structure in the outer disk, coincident with the optical spiral arms. Only in the high sensitivity $\mathrm{CnD}$ data is the velocity field at the center of NGC 1398 detected. A high resolution image (Figure 1) reveals small irregularities where the spiral structure is crossed but in general the velocity field is symmetric and well ordered. Contours at either end of the major axis close, indicating a falling rotation curve. The velocity field is used to determine orientation parameters and a galactic rotation curve. Solutions are stable over a range of radii and opening angles and between the receding and approaching halves of the galaxy. From the rotation curve we calculate the angular velocity and attempt to determine the pattern speed of the galaxy.

NGC 1398 has several morphological features thought to mark major resonances. The inner ring is expected to be the signature of either the inner $4 / 1$ resonance (Schwarz 1984; Byrd et al. 1994) or of corotation (Contopoulos \& Grosbøl 1989; Kaufmann 1992). The outer double pseudorings mark the OLR (Buta 1995). Using the ring structures to indicate possible resonances, several kinematic models are explored. Single pattern models that place the inner ring at either the inner $4 / 1$ resonance or corotation give results in contradiction to both numerical simulations and stellar orbit calculations. A model which places 

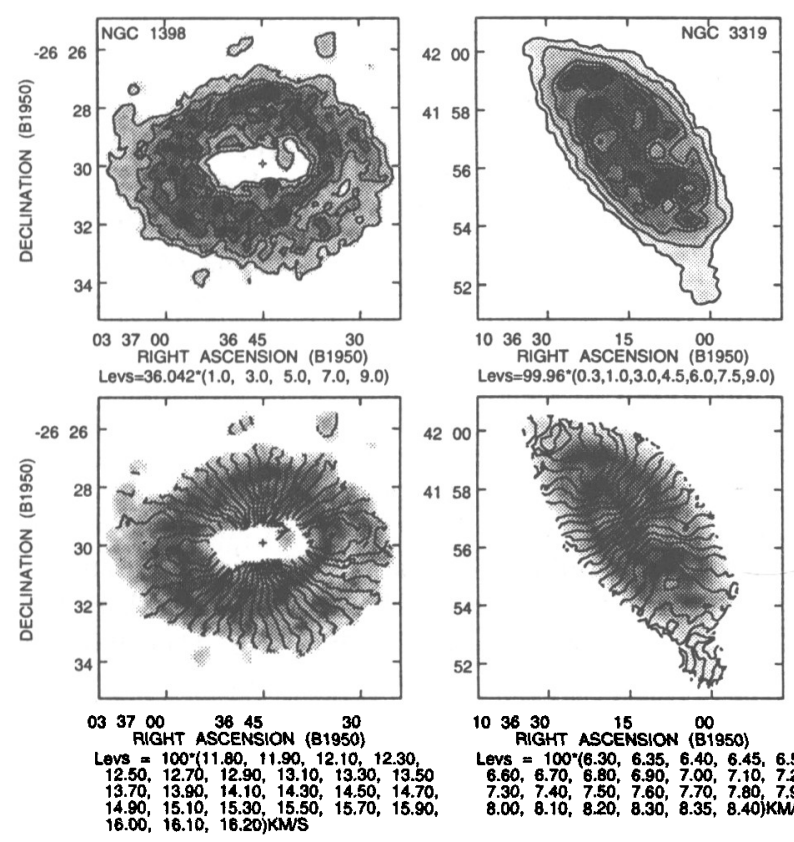

Levs $=100^{*}(6.30,6.35,6.40,6.45,6.50$,

$6.60,6.70,6.80,6.90,7.00,7.10,7.20$, $7.30,7.40,7.50,7.60,7.70,7.60,7.90$,

Figure 1. The HI surface density and velocity fields of NGC 1398 (left) and NGC 3319 (right). All images were made from combined (i.e., C plus D arrays) data sets. North is up and east is to the left.

the OLR at the average radius of the two outer pseudorings as suggested for $R_{1} R_{2}^{\prime}$ systems matches the data fairly well and gives results in accord with theory. It is not clear that the $R_{1} R_{2}^{\prime}$ classification is correct however. Optical images seem to show a continuous spiral, a not pseudoring structure. We also consider a two pattern model (Sellwood \& Sparke 1988). Corotation of the inner pattern is placed at the ring radius. The OLR of this pattern is the corotation of the outer pattern and serves as a "groove" which generates both inward and outward traveling spiral density waves. This fits the observations well. The OLR of the outer pattern lies beyond the optical spiral structure and the inward propagating spirals do not end at any preferred angle with respect to the bar, both points which support this model.

\section{NGC 3319}

NGC 3319 was observed with the D and C arrays of the VLA in 1993. A channel separation of $10.33 \mathrm{~km} \mathrm{~s}^{-1}$ was obtained with spatial resolutions from 10 " to 50 ". Details of the observations are given in Moore \& Gottesman (1995b). The global HI distribution (Figure 1) shows a narrow gas bar and three spiral features, two in the north, one in the south. The spiral structure is distorted in the south, most likely by the low density regions, the largest of which is clearly seen on the western side of the galaxy. Kinematic evidence suggests these are shells or 
supershells caused by supernovae explosions. The HI distribution is also strongly asymmetric. In a D array image, the galaxy extends 100 " further in the south, out to $400 "$, due to the presence of an off center tail.

The radial velocity field of NGC 3319 (Figure 1) shows irregularities due to the bar, low density regions and spiral structure, complicating the rotation curve analysis. The position angle of the line of nodes and the inclination show large deviations due to these structures. The variations are attributed to small scale structure rather than to a warp within a radius of 200". Beyond 200" however, large deviations are present in the velocity field in the south. It is not clear what is causing these or the HI tail. NGC 3319 is an isolated galaxy and neither tidal distortions nor a recent merger seem likely. The outer disk may be warped but it does not show the normal integral sign shape but rather bends in the same direction on both halves of the galaxy. Assuming the gas disk within $190^{\prime \prime}$ is planar and thus global values of the position angle and inclination are appropriate, a rotation curve is determined. Using a kinematic technique to determine the corotation radius (Canzian 1993) yields no conclusive results. However, Zaritsky, Kennicutt \& Huchra (1994) find a break in the abundance gradient of NGC 3319 at a radius between 40 to 70 " (the bar radius is 50 "). Friedli \& Benz (1995) show that such breaks are indications of the corotation radius and of a young bar. HII regions along the bar also imply a young bar.

NGC 3319 , a late type galaxy, appears to be a dynamically young system. Rich in central HI, the dynamical effects of the bar have not had time to sweep out the gas, in contrast to the early system, NGC 1398. NGC 1398 is a dynamically more advanced system with a large bar and bulge combination, a reduced rate of star formation, and more regular spiral structure. The contrast between the well organized, highly regular form of NGC 1398 and the irregular form and perturbed velocity field of NGC 3319 are most likely due to their different stages of dynamical evolution.

\section{References}

Buta, R. 1995, ApJS, 96, 39

Byrd, G., Rautiainen, P., Salo, H., Buta, R., \& Crocker, D. A. 1994, ApJ, 108, 476

Canzian, B. 1993, ApJ, 414

Contopoulos, G. \& Grosbø1, P. 1989 A\&A Rev., 1, 261

Friedli, D. \& Benz, W. 1995, A\&A, 301, 649

Kaufmann, D. E. 1992, in Astrophysical Disks, Dermott, S. F., Hunter, J. H., \& Wilson, R. E., 675, 140.

Moore, E. M. \& Gottesman, S. T. 1995a, ApJ, 447, 159

Moore, E. M. \& Gottesman, S. T. 1995b, ApJ, submitted

Schwarz, M. P. 1984. MNRAS, 209, 93

Sellwood, J. A. \& Sparke, L. S. 1988, MNRAS, 231, 25p

Zaritsky, D., Kennicutt, R. C. Jr., \& Huchra, J. P. 1994, ApJ, 420, 87 